\title{
THREE-DIMENSIONAL VISUALIZATION OF TEETH BY MAGNETIC RESONANCE IMAGING DURING SPEECH
}

\author{
Sandra R. Ventura (1), Diamantino R. Freitas (2), Isabel M. Ramos (3), \\ João Manuel R. S. Tavares (4)
}

\begin{abstract}
1. Área Técnico-Científica da Radiologia, Escola Superior de Tecnologia da SaúdeInstituto Politécnico do Porto, Portugal, sandra.rua@eu.ipp.pt;

2. Departamento de Engenharia Electrotécnica e de Computadores, Faculdade de Engenharia, Universidade do Porto,Portugal, dfreitas@fe.up.pt;

3. Departamento de Radiologia do Centro Hospitalar de S. João EPE, Faculdade de Medicina, Universidade do Porto, Portugal, radiologia.hsj@mail.telepac.pt;

4. Instituto de Engenharia Mecânica e Gestão Industrial, Departamento de Engenharia Mecânica, Faculdade de Engenharia, Universidade do Porto, Portugal, tavares@fe.up.pt
\end{abstract}

\section{Introduction}

Teeth morphology and oral cavity visualization through imaging techniques enables an accurate vocal tract assessment in speech research, especially when by Magnetic Resonance Imaging (MRI) [1].

Magnetic Resonance Imaging has been successfully applied on real-time analysis of the articulators during speech production, along the whole vocal tract, with good signal-to-noise ratio and without ionizing effects. As such, several MRI techniques provided the calculation of cross-sectional areas and volumes directly from static postures during sustained articulations [2] or using multiple repetitions [3, 4].

Currently, the application of MRI in speech research provides useful and accurate qualitative and quantitative analysis of speech articulation [5]. The role of teeth during speech is important not only to the evaluation of dental and lingual sounds, but also for measuring the vocal tract shape in three dimensions (3D) and the mouth acoustic opening [6].

The vocal tract is similar to an inverted L-shaped tube, with a significant length, which extends from the vocal folds to the lips and with a side branch leading to the nasal cavity [7] and nostrils. The articulators include the lips, the tongue, the teeth, the palate and the pharynx, and establish a set of cavities for air stream modification during speech. Because of the wide range of movements and flexibility of some articulators, in particularly of the tongue, it is difficult to observe and assess their movements and deformations.

Some methods for teeth observation have been attempted intending the qualitative and quantitative analysis of speech articulation using different techniques $[1,8]$, mainly using MRI; however, with some drawbacks, especially for speech production assessment purposes, as summarized in Tables 1 and 2 .

\begin{tabular}{|c|c|}
\hline Study & Purpose and drawbacks \\
\hline $\begin{array}{l}\text { Wakumoto } \\
\text { et al., } 1997 \\
\text { [cited in } 9 \text {, } \\
10]\end{array}$ & $\begin{array}{l}1^{\text {st }} \text { trial performed using MRI. } \\
\text { Paired plates containing contrast medium to } \\
\text { visualize dental crown. } \\
\text { Image-by-image manual segmentation of teeth } \\
\text { boundaries. } \\
\text { Indirect visualization of teeth crowns. Cannot } \\
\text { be used during speech articulation, especially } \\
\text { of some fricative consonants. }\end{array}$ \\
\hline $\begin{array}{l}\text { Tutton \& } \\
\text { Goddard, } \\
2002 \text { [12] }\end{array}$ & $\begin{array}{l}\text { MRI appearances of the anatomy of pathology } \\
\text { of the dento-alveolar region. } \\
\text { Reveals that the best technique for } \\
\text { demonstrating the crowns of the teeth is with } \\
\text { the subject with his mouth close and refrain } \\
\text { swallowing during the scan. } \\
\text { Cannot be used during speech articulation. }\end{array}$ \\
\hline $\begin{array}{c}\text { Olt \& } \\
\text { Jakob, } \\
2004[8]\end{array}$ & $\begin{array}{l}\text { Contrast-enhanced dental MRI for 3D } \\
\text { visualization of the oral cavity, jaw and teeth. } \\
\text { Pure water was used as contrast medium inside } \\
\text { the oral cavity. } \\
\text { Isosurface reconstructions (3D) of the teeth and } \\
\text { jaw were performed. } \\
\text { Indirect visualization of teeth crowns. Subject } \\
\text { effort and discomfort; artifacts can occur. } \\
\text { Cannot be used during speech articulation. }\end{array}$ \\
\hline $\begin{array}{c}\text { Takemoto } \\
\text { et al., 2004 } \\
{[1]}\end{array}$ & $\begin{array}{l}\text { Three steps: } 1^{\text {st }} \text { dental scans for extracting } \\
\text { digital jaw casts with the subject holding } \\
\text { blueberry juice inside the mouth; } 2^{\text {nd }} \text { the } \\
\text { superimposition of teeth in the images } \\
\text { collected during speech based on the position } \\
\text { matching of the reference landmarks; } 3^{\text {rd }} \\
\text { extraction of 3D vocal tract shape. } \\
\text { Some errors in landmark sampling, due to the } \\
\text { different body positions of the subject (prone } \\
\text { position during the } 1^{\text {st }} \text { scan and then supine } \\
\text { position for speech production scans) and } \\
\text { motion. } \\
\text { Indirect visualization of teeth crowns. Subject } \\
\text { effort and discomfort due to long acquisition } \\
\text { times. }\end{array}$ \\
\hline
\end{tabular}


Table 1. Current methods concerning the teeth visualization using MRI (to continue).

\begin{tabular}{|c|c|}
\hline Study & Purpose and drawbacks \\
\hline $\begin{array}{l}\text { Ventura et al., } \\
2009[5]\end{array}$ & $\begin{array}{l}\text { Two steps: } 1^{\text {st }} \text { an acquisition of a midsagittal } \\
\text { high resolution image with the tongue in full } \\
\text { contact with the tooth for manual extraction of } \\
\text { teeth shape; } 2^{\text {nd }} \text { superimposition of tooth in } \\
\text { further sagittal images collected during speech } \\
\text { production using manual editing and pasting. } \\
\text { Misalignments errors and time consuming } \\
\text { method. } \\
\text { Indirect visualization of incisors crowns. } \\
\text { Not possible to obtain 3D models of teeth due to } \\
\text { reduced volume image data. }\end{array}$ \\
\hline $\begin{array}{l}\text { Idiyatullin et } \\
\text { al., } 2011 \text { [13] }\end{array}$ & $\begin{array}{l}\text { In vitro and in vivo teeth MRI to assess a recent } \\
\text { technique - Sweep Imaging with Fourier } \\
\text { Transform (SWIFT). } \\
\text { Images acquired using a 9.4T and } 4.0 \mathrm{~T} \text { systems } \\
\text { and further compared with other techniques. } \\
\text { Offers simultaneous 3D hard and soft tissues } \\
\text { imaging of the teeth. } \\
\text { High cost of SWIFT-based MRI systems. } \\
\text { Low affordability of MRI systems and ultra- } \\
\text { high fields not approved for patient clinical } \\
\text { management. } \\
\text { Cannot be used during speech articulation due to } \\
\text { long acquisition times. }\end{array}$ \\
\hline $\begin{array}{l}\text { Kitamura et } \\
\text { al., } 2011 \text { [11] }\end{array}$ & $\begin{array}{l}\text { Measurement of the tooth shape separately from } \\
\text { MRI data acquisition during speech production. } \\
\text { Thermoplastic elastomer dental mouthpiece } \\
\text { used to measure the 3D tooth shape. } \\
\text { The proposed method requires two MRI } \\
\text { acquisitions and the correction of the chemical } \\
\text { shift artifact to reconstruct the volume data. } \\
\text { Imposes less physical strains on subjects. } \\
\text { Cannot be used during speech articulation. }\end{array}$ \\
\hline $\begin{array}{c}\mathrm{Ng} \text { et al., } 2011 \\
{[6]}\end{array}$ & $\begin{array}{l}\text { A custom-made retainer filled with the jelly } \\
\text { form of ferric ammonium citrate was used for } \\
\text { superimposition of the incisor boundary into } \\
\text { sequential images of MRI movie. } \\
\text { Landmarks on the forehead, nasal root, nasal tip } \\
\text { and points along the cranium were used for } \\
\text { superimposition of the upper incisor. } \\
\text { Direct visualization of incisors crowns. } \\
\text { Detailed geometry relationships between tongue } \\
\text { and tooth were observed during the production } \\
\text { of some consonants. } \\
\text { Not possible to obtain 3D models of teeth. }\end{array}$ \\
\hline
\end{tabular}

Table 2. Current methods concerning the teeth visualization using MRI (conclusion).

As found during the literature review, teeth visualization by MRI is growing and in different directions, being the results promising, not only for dentistry and orthodontics, but also for speech research purpose.

The aim of this study was to describe and present an effective and useful method to extract the teeth morphology in MRI images that facilitates the MRI based qualitative and quantitative analysis concerning speech articulation.

\section{Material and methods}

In this section, the procedures adopted to visualize teeth using MR images are described. Thus, the MRI equipment and protocol adopted in the acquisition process is specified; afterwards, the image data processing and analysis accomplished is described.

\subsection{Teeth visualization}

During this study, some experiments, including one similar to the one described in [1], were attained to improve teeth visualization using MRI; however, due to the discomfort implied to the subjects and the indirect visualization of the dental crows, these experiments were discarded.

Due to the lack of signal from teeth produced by MRI and considering only the methods that could be possible to use during speech, we pursued the use of materials with the following requirements:

- Biocompatibility in oral cavity, easy and effective to use;

- Biosafety in MRI environment;

- High intensity on conventional brain MR images, especially T1-weighted images using either spin echo or gradient-echo sequences;

- Possible to use during phonation without affect the speech articulation;

- Availability and affordability of the material.

For this purpose, with the collaboration of a medical dentist, four solutions were tested although without the expected results: 1) fluorine coating, (2) orthodontic adhesive, (3) plastic mouthpiece coating with whitening gel and (4) thin-layer (of 2 $\mathrm{mm}$ ) molded silicone mouthpiece for the upper jaw. Considering the good adhesion of the silicone mold to the teeth and because it is subject custom-made, we decided to improve this solution. Therefore, after some experiments, the new solution was to coat the molded silicone mouthpiece of the upper jaw with a thin-layer of petroleum jelly. This substance was chosen because it is a hydrophobic hydrocarbon, is insoluble in water, provides high intensity on T1-weighted images and is an effective oral contrast. The silicone mouthpiece developed can be seen in Figure 1.

Cod liver oil capsules were used as head surface landmarks in order to facilitate the localization of the molded silicone mouthpiece along the acquired volumetric MRI data for the subsequent image processing and analysis.

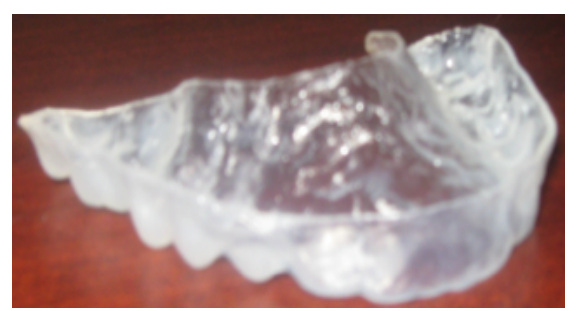

Figure 1.The molded silicone mouthpiece of the upper jaw coated with a thin-layer of petroleum jelly. 


\subsection{MR system and protocol imaging}

The MRI data was acquired using a clinical 3.0 Tesla MRI system at the Radiology Department of the Hospital S. João, Porto, Portugal. Experiments were performed with one female subject during some speech production tasks and according to the specific safety procedures.

The first images were acquired using a turbo spin echo 2D sequence, and consisted of one T1weighted $4 \mathrm{~mm}$ thick midsagittal slice. Two tasks were performed during the image acquisition with the subject in a supine position: 1) with the mouth in rest position and then 2) demand to sustain the sound /a/. The remainder imaging acquisition parameters adopted were the following: a repetition time of $400 \mathrm{~ms}$, an echo time of $10 \mathrm{~ms}$, an echo train length of 5, a square field of view of $240 \mathrm{~mm}$, a matrix size of $512 \times 512$ pixels, a resolution of 2.133 pixels per $\mathrm{mm}$ and a pixel size of $0.469 \times 0.469 \mathrm{~mm}$. The acquisition time lasted around 8.07 seconds for each task.

Afterwards, a 3D volumetric MRI of the vocal tract resonance cavities was carried out using a flash gradient echo sequence during the speech task. A single $120 \mathrm{~mm}$ thick midsagittal slab was acquired according to the following parameters: repetition time of $5.8 \mathrm{~ms}$, echo time of $2.17 \mathrm{~ms}$, flip angle equal to $10^{\circ}$, a square field of view of $270 \mathrm{~mm}$, a matrix size of $256 \times 256$ pixels, a resolution of 0.948 pixels per $\mathrm{mm}$ and a pixel size of $1.05 \times 1.05 \mathrm{~mm}$. The acquisition time lasted around 16.12 seconds.

\subsection{Image processing and analysis}

The image-based reconstruction of the 3D dental shapes was carried out using the ITK-SNAP software (version 2.1.4-rc1) developed by the Penn Image Computing and Science Laboratory (PICSL). The 3D models of the teeth were automatically built using snakes to segment each slice and posterior interpolation of the resultant contours.

\section{Results and discussion}

By means of this MRI-based, method the entire vocal tract was imaged resulting in two sagittal slices that permit to visualize the teeth boundaries. Figure 2 depicts the images acquired from the subject with the mouth in rest position, i.e. mouth and lips closed, and during the production of the sound $/ \mathrm{a} /$.
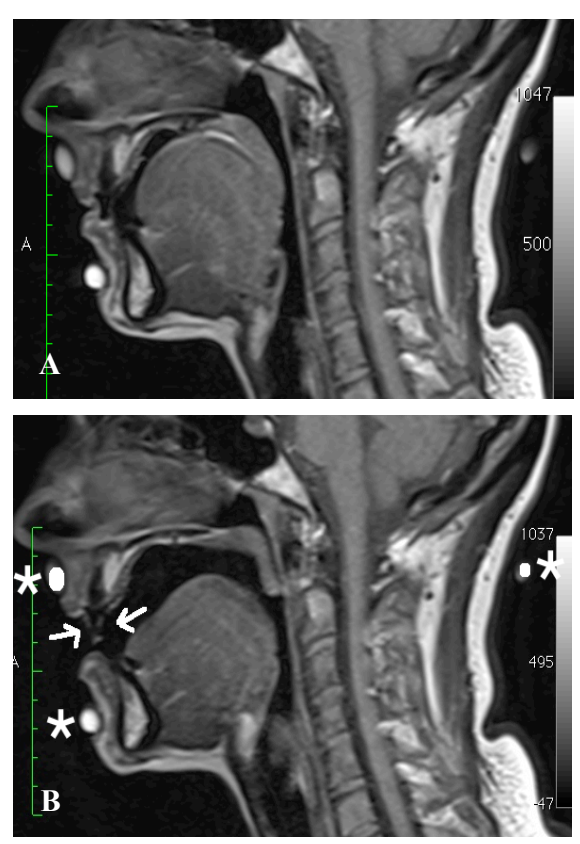

Figure 2. MR T1-weighted images depicting the teeth boundaries (indicated by arrows) and cod liver oil capsules used as head surface landmarks (indicated by asterisks), with mouth according to the rest position (A) and during the speech production (B).

As can be observed in Figure 2, the teeth boundaries are clearly visible during the speech production.

From the 3D volumetric MRI data acquired, a total of 60 transversal images corresponding to slices with $2 \mathrm{~mm}$ of thickness was analysed. The image processing and reconstruction results are depicted in Figure 3. The display windows included show the segmented dental area and the 3D models built for the teeth.
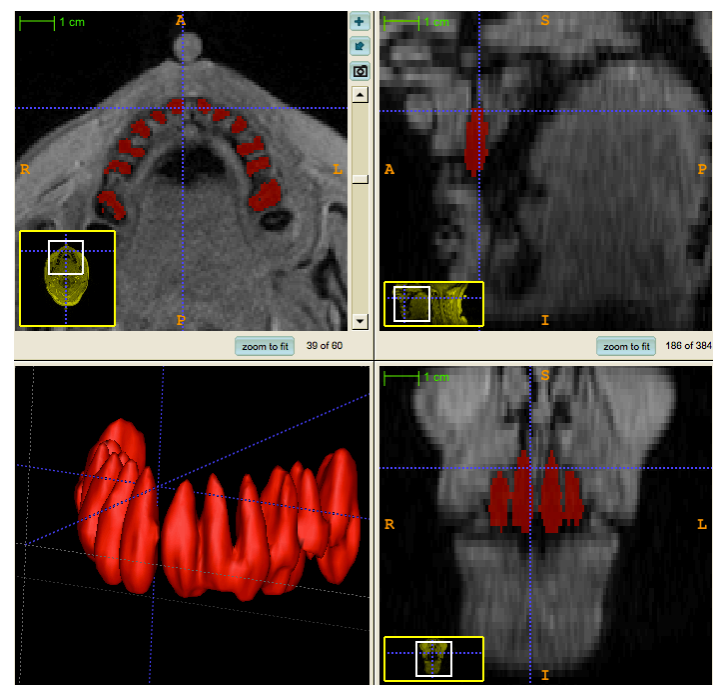

Figure 3. Image processing and $3 \mathrm{D}$ reconstruction of teeth from volumetric MRI data accomplished using the ITK-SNAP software. 
In addition, the study was extended to the relationships among the tongue, the teeth and the oral cavity during speech production. The 3D models built for the tongue, the teeth and the oral cavity are shown in Figure 4.
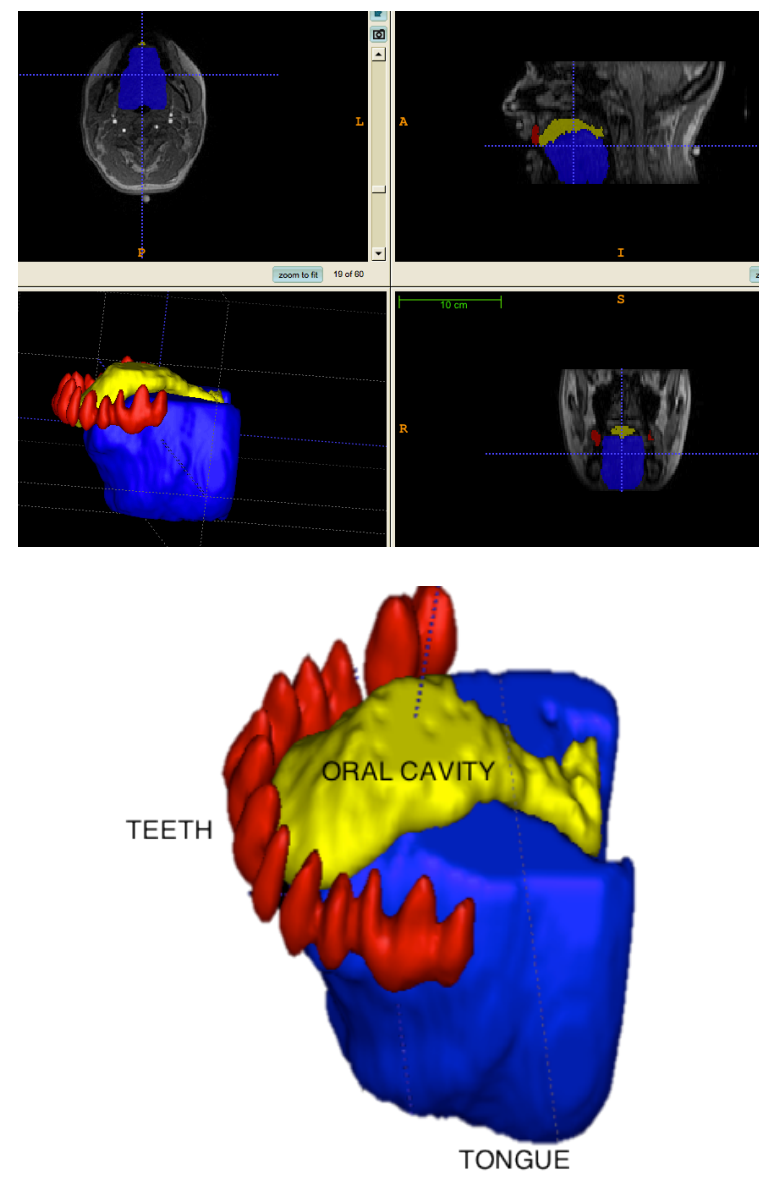

Figure 4. Image processing and $3 \mathrm{D}$ reconstruction of the teeth, tongue and oral cavity from volumetric MRI data accomplished using the ITK-SNAP software.

\section{Conclusions}

Our approach allows the visualization of the dental morphology and the extraction of 3D information related with speech articulators, for speech research purposes.

The proposed approach allows the visualization of the teeth in both static and dynamic MRI acquisitions during speech production; in addition, it is feasible and affordable.

Through the present development, volumetric MRI can be successfully applied to obtain 3D models, and measurable data concerning the vocal tract resonance cavities and all the articulators involved, including for the first time the teeth. This knowledge is especially important for speech studies based on MRI.

As future work, more experiments in different subjects must be addressed in order to evaluate spatial and temporal relationships among articulators during speech.

The proposed approach may also be effective and easy to apply in other medical fields such as dentistry and orthodontics.

\section{Acknowledgments}

The authors would like to express their gratitude to Dr Augusta Marques, from Arrábida Hospital, Vila Nova de Gaia, in Portugal, for her kind collaboration.

The images used in this work were acquired at the Radiology Department of Hospital S. João EPE, in Portugal. The authors would like to express their gratitude to the Hospital technical staff.

The first author would like to thank the support and contribution of the $\mathrm{PhD}$ grant from Escola Superior de Tecnologia da Saúde (ESTSP) and Instituto Politécnico do Porto (IPP), in Portugal.

This work was partially done in the scope of the project "Methodologies to Analyze Organs from Complex Medical Images - Applications to Female Pelvic Cavity", with reference PTDC/EEACRO/103320/2008, financially supported by Fundação para a Ciência e a Tecnologia, in Portugal.

\section{References}

[1] Takemoto, T., Kitamura, T., Nishimoto, H., Honda, K., A method of tooth superimposition on MRI data for accurate measurement of vocal tract shape and dimensions, Acoustical Sciences \& Technology, 25(6): 468-474, 2004.

[2] Kim, Y-C., Narayanan, S.S., Nayak, K.S., Accelerated Three-Dimensional Upper Airway MRI Using Compressed Sensing, Magnetic Resonance in Medicine, 61: 1434-1440, 2009.

[3] Stone, M., Davis, E.P. Douglas, A.S., NessAiver, M., Gullapalli, R., Levine, W.S., Lundberg, A., Modeling the motion of the internal tongue from tagged cine-MRI images, Journal of the Acoustical Society of America 109(6): 29742982, 2001.

[4] Shadle, C.H., Mohammad, M., Carter, J.N., Jackson, P.J.B., Multi-planar Dynamic Magnetic Resonance Imaging: New Tools for Speech Research. In International Congress of Phonetics Sciences (ICPhS99), San Francisco, 623-626, 1999. [5] Ventura, S.R., Freitas, D.R., Tavares, J.M., Application of MRI and biomedical engineering in speech production study, Computer Methods in Biomechanics and Biomedical Engineering, 12(6): 671-681, 2009.

[6] Ng, I.W., Ono, T., Inoue-Arai, M.S., Honda, E., Kurabayashi, T., Moriyama, K., Application of MRI movie for observation of articulatory 
movement during fricative /s/ and a plosive /t/, Angle Orthodontist, 81(2): 237-244, 2011.

[7] Ventura, S.R., Freitas, D.R., Tavares, J.M., Toward Dynamic Magnetic Resonance Imaging of the Vocal Tract During Speech Production, Journal of Voice, 25(4): 511-518, 2011.

[8] Olt, S. \& Jakob, P.M., Contrast-Enhanced Dental MRI for visualization of the Teeth and Jaw, Magnetic Resonance in Medicine, 52:174-176, 2004.

[9] Masaki, S., Nota, Y., Takano, S. Takemoto, H., Kitamura, T., Honda, K., Integrated magnetic resonance imaging methods for speech science and technology, The Journal of the Acoustical Society of America, 123(5): 5083-5088, 2008.

[10] Kitamura, T., Nishimoto, H., Fujimoto, I., Shimada, Y., Dental imaging using magnetic resonance visible mouthpiece for measurement of vocal tract shape and dimensions, Acoustical Sciences \& Technology, 32(5): 224-227, 2011.

[11] Tutton, L.M. \& Goddard, P.R., MRI of the teeth, The British Journal of Radiology, 75: 552562, 2002.

[13] Idiyatullin, D., Corum, C., Moeller, S., Prasad, H.S., Garwood, M., Nixdorf, D.R., Dental magnetic resonance imaging: making the invisible visible, Journal of Endodontics, 37(6): 745-752, 2011. 